Check for updates

Cite this: RSC Adv., 2019, 9, 24646

Received 17th May 2019

Accepted 25th July 2019

DOI: 10.1039/c9ra03728d

rsc.li/rsc-advances

\title{
Covalently linked benzimidazole-containing reduced graphene oxide/polyaniline nanocomposites as electrode materials $\uparrow$
}

\begin{abstract}
Arkapal Roy, Saptarshi Dhibar, Sibu Kundu and Sudip Malik (D) *
In the present manuscript, we reported the effective synthesis of 1,3-bis(2'-benzimidazolyl)-5aminobenzene-grafted graphene oxide, followed by reduction, affording covalently linked benzimidazole-containing chemically modified reduced graphene oxide. The prepared material was further coated with polyaniline via in situ polymerization. The prepared material was successfully characterized by Fourier transform infrared (FT-IR) spectroscopy, X-ray powder diffraction (XRPD), X-ray photoelectron spectroscopy (XPS), field emission scanning electron microscopy (FESEM), transmission electron microscopy (TEM), and thermo-gravimetric analysis (TGA); subsequently, it was subjected to electrochemical analysis using a three-electrode system in dilute acid solutions as electrolytes in terms of cyclic voltammetry (CV), galvanostatic charge-discharge and electrochemical impedance studies. The polyaniline-coated binary composite materials revealed maximum specific capacitance of $823 \mathrm{~F} \mathrm{~g}^{-1}$ at $0.2 \mathrm{~A} \mathrm{~g}^{-1}$ current density, whereas the value for the chemically modified reduced graphene oxide was $477 \mathrm{~F} \mathrm{~g}^{-1}$. The CV curves denoted the pseudocapacitive nature of the electrode materials. The values for the retention of specific capacitance for the electrode materials were $77.5 \%$ and $87.9 \%$ up to 5000 charge-discharge cycles.
\end{abstract}

\section{Introduction}

Carbon-based materials are realized as supreme electrode materials for energy generation as well as storage due to their high electrical conductivity, good structural firmness and easy availability, considering the escalated distress regarding global warming and the crunch in the source of fossil fuels. Directly, a feasible energy source maintaining efficient energy and storage technology is urgently needed. ${ }^{1-4}$ In the era of the $21^{\text {st }}$ century, one of (consequently) the most important targets facing the scientific community is to fabricate materials in a traditional way via a green and low-cost approach, meeting the demand of sustainable energy sources. Among the carbonbased materials, graphene is one of the leading components with attractive properties such as a high surface area, large electrical conductivity, unique heterogeneous electron transfer and charge carrier rates $^{5-7}$ with wide applications in electrochemical fields such as energy storage ${ }^{8-10}$ in the form of capacitors. Electrochemical capacitors, also named as

School of Applied and Interdisciplinary Sciences, Indian Association for the Cultivation of Science, $2 A$ and $2 B$ Raja S. C. Mullick Road, Jadavpur, Kolkata-700032, India. E-mail: psusm2@iacs.res.in

$\dagger$ Electronic supplementary information (ESI) available: NMR and FT-IR characterization data of 1,3-bis(2'-benzimidazolyl)-5-aminobenzene, XRD of RGONBZ_PANI, FESEM image, XPS of GO and different electrochemical plots of different sets of binary composite materials. See DOI: 10.1039/c9ra03728d supercapacitors or ultracapacitors, have attracted remarkable attention as promising energy storage systems owing to their high power density, reversibility, high rate capability and longterm durability with low maintenance. According to their charge storage mechanism, capacitors can be classified into electrochemical double-layer capacitors (EDLC) and pseudocapacitors. EDLCs perform depending on the electrostatic interactions between the ions in the electrolyte and the active electrode materials, whereas pseudo-capacitors store and release energy by fast surface faradaic redox reactions. ${ }^{1}$ Pseudocapacitors have much higher capacitance than EDLCs although they exhibit short capacitance retention because the redox reactions are not entirely reversible and the structural damage during the redox process limits their industrial applications. ${ }^{\mathbf{1 1}}$ Thus, it is more important to construct electrochemical capacitors with higher capacitance having excellent cyclic stability.

Graphene, an allotrope of carbon in a two-dimensional sheet-like form, has attracted great attention owing to its high surface area, low mass density, and excellent mechanical properties and conductivity, due to which it is a rapidly rising star on the horizon of materials science. ${ }^{12}$ Graphene oxide (GO) is a single sheet of graphite oxide carrying different oxygencontaining functional groups such as hydroxyl and epoxy groups bound on the basal planes in addition to the partial carboxyl groups located at the edges. ${ }^{13}$ Unfortunately, the GO sheets tend to either form irreversible agglomerates or restack 
during the solution reduction process, resulting in the decrease in surface area, a dominant factor in the supercapacitor application. ${ }^{14,15}$ Many viable active species such as conducting polymers, heterocyclic moieties and transition metal oxides have been introduced to inhibit the restacking of graphene sheets as well as to improve the capacitance performance. However, they may suffer from the cyclic retention of specific capacitance because of their volume change and ion dissolution during the charge-discharge processes. ${ }^{16-18}$ Covalently functionalized graphene oxides have redox-active functional groups and they exhibit an enhanced capacitance performance. These rapidly occurring redox reactions at the electrode surface may elevate the charge storage capability. ${ }^{17}$ Yongsheng Chen et al. synthesized $\mathrm{N}$-doped graphene materials using $o$-phenylenediamine as the double-N dopant by an easy solvothermal process, which showed specific capacitance up to $301 \mathrm{~F} \mathrm{~g}^{-1}$ with excellent cycling stability. ${ }^{19}$ Wei Huang et al. reported the use of benzoxazole- and benzimidazole-grafted reduced graphene oxides as pseudocapacitors, showing specific capacitances up to $730 \mathrm{~F}$ $\mathrm{g}^{-1}$ and $781 \mathrm{~F} \mathrm{~g}^{-1}$, respectively. ${ }^{20}$

PANI has received much attention because of its easy synthesis, highly controllable conductivity, good environmental friendliness, and increased capacitance from multiple redox states in comparison to those of other polymer networks such as poly(3,4-ethylenedioxythiophene)s (PEDOTs), polythiophenes (PThs), and polypyrroles (PPys). Thus, graphene- and PANIcontaining composite materials should exhibit improvement in the conductivity of PANI as well as decrease in the aggregation of graphene materials via the synergistic effect. Till now, numerous reports regarding different synthetic methods such as electrochemical synthesis ${ }^{21,22}$ and chemical in situ polymerization are available for graphene-PANI composites. ${ }^{23,24}$ Wang $\mathrm{R}$. et al. reported the hydrothermal synthesis of nanostructured PANI composited with graphene sheets in different morphologies as an electrode material for supercapacitors. ${ }^{25} \mathrm{Xu}$ et al. prepared a nanocomposite where PANI nanoparticles were uniformly coated on GO sheets with the assistance of supercritical carbon dioxide and it exhibited specific capacitance of $425 \mathrm{~F} \mathrm{~g}^{-1}$ at the current density $0.2 \mathrm{~A} \mathrm{~g}^{-1}{ }^{-26}$ Liu et al. described another kind of carboxyl-functionalized graphene oxide and PANI nanocomposite as a promising supercapacitor electrode material. ${ }^{27}$ To the best of our knowledge, there is no report available on $\mathrm{N}$-doped graphene oxide containing a benzimidazole moiety and a PANI nanocomposite as an electrode material.

Herein, we reported the synthesis of 1,3 -bis $\left(2^{\prime}\right.$ benzimidazolyl)-5-aminobenzene-doped graphene oxides and PANI binary composites (Schemes 1 and 2) via in situ oxidative polymerization in an $\mathrm{HCl}$ medium with ammonium persulfate (APS) serving as an oxidizing agent. Furthermore, the prepared composites were utilized as electrode materials. Prior to the previous reaction, hydrothermal condensation was performed between graphene oxide and 1,3-bis(2'-benzimidazolyl)-5aminobenzene, followed by reduction using hydrazine monohydrate. The doped graphene oxides as well as the composite materials were characterized by FT-IR and XPS experiments and the morphology was properly investigated by FESEM and TEM experiments. The electrochemical performances were measured by cyclic voltammetry (CV), galvanostatic charge-discharge (GCD) and electrochemical impedance (EIS) analyses.

\section{Experimental sections}

\subsection{Materials}

Graphite powder (12 500, 500 mesh), 5-aminoisophthalic acid (5-AIPA), ortho-phenylenediamine (OPD), ammonium persulfate (APS) and polyphosphoric acid (reagent grade, 115\% $\mathrm{H}_{3} \mathrm{PO}_{4}$ ) were purchased from Sigma-Aldrich and used without further purification. Aniline (distilled under reduced pressure and stored at $5{ }^{\circ} \mathrm{C}$ in a dark place before use), sodium bicarbonate $\left(\mathrm{NaHCO}_{3}\right)$, potassium permanganate $\left(\mathrm{KMnO}_{4}\right)$, sodium nitrate $\left(\mathrm{NaNO}_{3}\right)$, hydrochloric acid $(\mathrm{HCl}, 37 \%)$, sulfuric acid $(98 \%)$, and hydrogen peroxide $(30 \% \mathrm{v} / \mathrm{v})$ were purchased from Merck Chemicals as analytically pure reagents. Herein, $10 \%(\mathrm{w} /$ v) $\mathrm{NaHCO}_{3}$ solutions were prepared with deionised water (18 $\mathrm{M} \Omega \mathrm{cm}$, Millipore Milli $\mathrm{Q}$ water system).

\subsection{Instrumentation}

The ${ }^{1} \mathrm{H}-\mathrm{NMR}$ spectrum of 1,3-bis(2'-benzimidazolyl)-5aminobenzene was recorded using a $500 \mathrm{MHz}$ Bruker DPX spectrometer at a MAS frequency of $10 \mathrm{kHz}$ and all chemical shifts were quoted using the $\delta$ scale (ppm). Fourier transform infrared (FT-IR) spectrum of $\mathrm{KBr}$ powder-pressed pellets was obtained using an FTIR-8400S instrument (Shimadzu). UV-vis spectroscopy of composite materials was performed using an Agilent (model 8453) UV-vis spectrophotometer. Thermogravimetric analysis of the polymer samples was performed using a TA SDT Q600 instrument at a heating rate of $10{ }^{\circ} \mathrm{C} \mathrm{min}^{-1}$ in a nitrogen atmosphere. X-ray powder diffraction (XRPD) analysis was performed using a Bruker AXS diffractometer (D8 advance) under $\mathrm{CuK} \alpha$ radiation $(\lambda=1.54 \AA)$ at a generator voltage of $40 \mathrm{kV}$ and a current of $40 \mathrm{~mA}$. XPS studies were performed using a focused monochromatized A1 K $\alpha$ X-ray source $(1486.8 \mathrm{eV})$ in an Omicron Nano-Technology 0571 XPS instrument. The surface morphology as well as the overall morphology of the composites was characterized using an FESEM (JEOL, JSM 6700F) instrument. To reduce the surface potential, samples were coated with platinum for $90 \mathrm{~s}$ prior to the experiment. TEM imaging was carried out using an HRTEM (JEOL, 2010EX) instrument at an accelerating voltage of $200 \mathrm{kV}$. A small amount of ethanol-dispersed samples was put over a 200 mesh $\mathrm{Cu}$ grid coated with a holey carbon support.

2.2.1 Electrochemical measurements. All electrochemical experiments like CV, GCD and electrochemical impedance were studied on a CHI6087 Electro-chemical Workstation (CHI, USA) using the conventional three-electrode system. A modified glassy carbon electrode (GCE, $3 \mathrm{~mm}$ in diameter with a surface area of $0.07 \mathrm{~cm}^{2}$ ) was used as the working electrode, a saturated calomel electrode (SCE) was used as the reference electrode, a Pt wire was used as the calomel electrode, and $1 \mathrm{M} \mathrm{H}_{2} \mathrm{SO}_{4}$ served as the electrolyte solution. For reuse, GCE was carefully polished with $1,0.3$, and $0.05 \mu \mathrm{m}$ alumina powder and sequentially washed with water and ethanol under sonication at room 


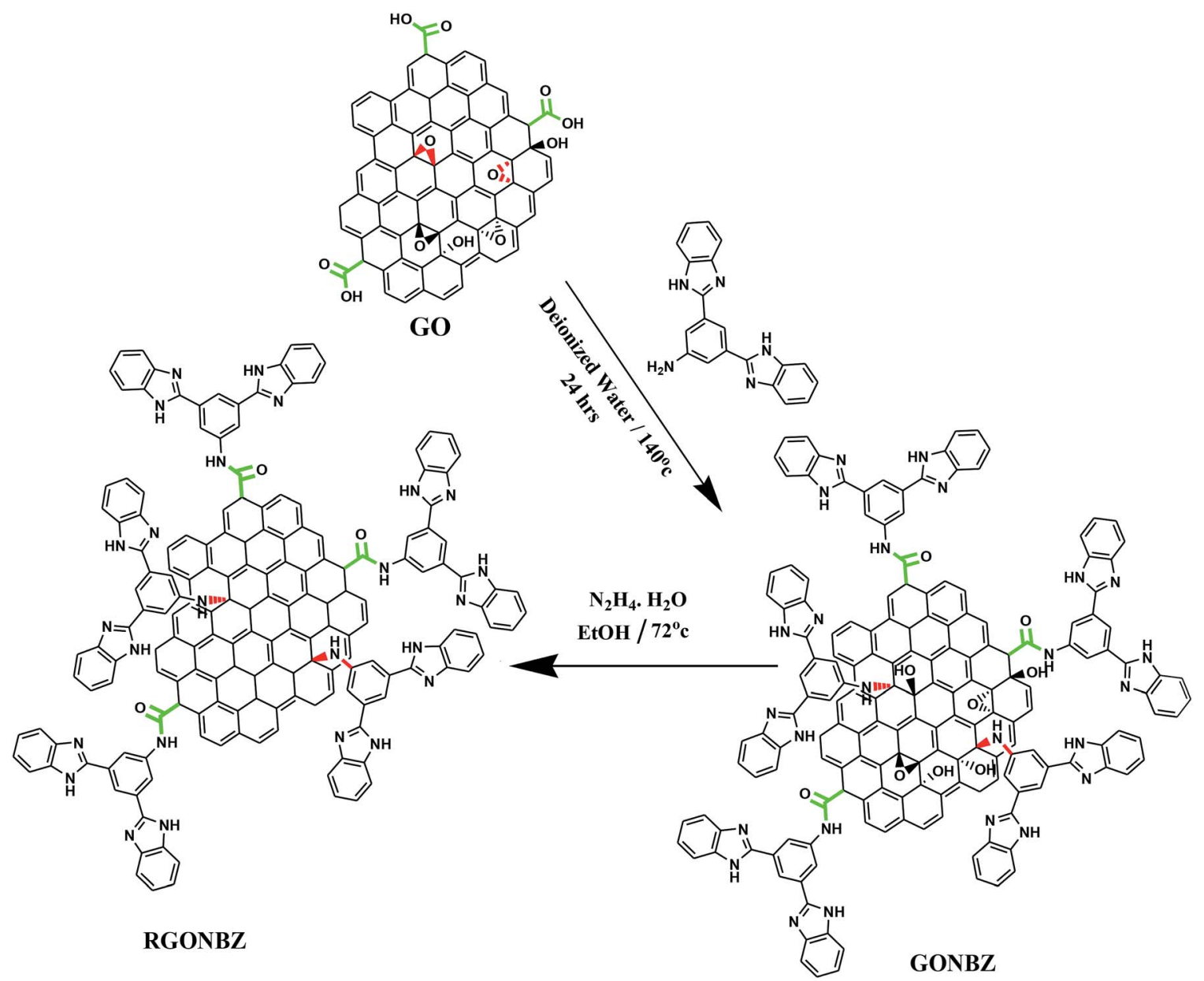

Scheme 1 Synthesis of 1,3-bis(2'-benzimidazolyl)-5-aminobenzene-grafted graphene oxide (GONBZ) and its reduced form (RGONBZ).

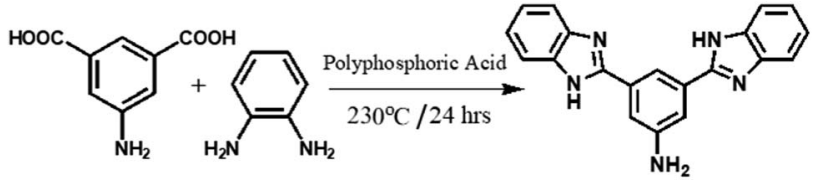

Scheme 2 Synthesis of 1,3-bis(2'-benzimidazolyl)-5-aminobenzene.

temperature until a mirror finish was obtained. The specific capacitance $\left(C_{\mathrm{s}}\right)$, energy density $(E)$ and power density $(P)$ were obtained from the discharge curve using the following three equations:

$$
\begin{aligned}
& C_{\mathrm{s}}=\frac{i \times \Delta t}{\Delta V \times m} \\
& E=\frac{C_{\mathrm{s}} \times \Delta V^{2}}{7.2} \\
& P=\frac{E \times 3600}{t}
\end{aligned}
$$

Here, $i=$ current, $m=$ mass of the polymer drop casted on the glassy carbon electrode surface, $\Delta t=$ discharge time, and $\Delta V=$ potential window.

\subsection{Synthesis of different derivatives}

The syntheses of 1,3-bis(2'-benzimidazolyl)-5-aminobenzene and $\mathrm{GO}$ were performed according to the procedure reported in the literature with slight modifications..$^{28,29}$

2.3.1 Synthesis of 1,3-bis( $2^{\prime}$-benzimidazolyl)-5-aminobenzene. A mixture of 5 -aminoisophthalic acid $(100 \mathrm{mg}, 0.55$ $\mathrm{mmol})$ and $o$-phenylenediamine $(150 \mathrm{mg}, 1.41 \mathrm{mmol})$ was taken in a $100 \mathrm{~mL}$ two-necked round-bottom flask (Scheme 2). First, it was made inert by $\mathrm{N}_{2}$ gas and $15 \mathrm{~mL}$ of polyphosphoric acid was added very fast, followed by heating the mixture at $230^{\circ} \mathrm{C}$ for $24 \mathrm{~h}$ in an inert atmosphere. The contents in the round-bottom flask were added to ice cold water $(300 \mathrm{~mL})$ with vigorous stirring under a slightly hot condition, resulting in a violet precipitate; it was collected by filtration and made acid-free by treating with $250 \mathrm{~mL} 10 \%$ warm $\mathrm{NaHCO}_{3}$ solution. Furthermore, the residue was dissolved in methanol and water was added to the methanol filtrate, resulting in an off-white precipitate, which was collected in a Buchner funnel and dried. The yield obtained was $85 \mathrm{mg}(48 \%)$.

${ }^{1} \mathrm{H}-\mathrm{NMR}\left(400 \mathrm{MHz}, \mathrm{d}_{6}\right.$-DMSO, TMS) $\delta(\mathrm{ppm}): 7.19(\mathrm{q}, 4 \mathrm{H})$; $7.482(\mathrm{~s}, 2 \mathrm{H}) ; 7.586$ (broad spectra, $4 \mathrm{H}) ; 8.132(\mathrm{~s}, 1 \mathrm{H})$; 12.947 (broad spectra, 2H). 
2.3.2 Synthesis of graphene oxide (GO). GO was synthesized from graphite powder by a modified Hummer's method. ${ }^{29}$ Briefly, $1 \mathrm{~g}$ graphite powder was slowly added to $50 \mathrm{~mL}$ conc. sulfuric acid taken in a $500 \mathrm{~mL}$ conical flask at $0-5{ }^{\circ} \mathrm{C}$ and stirred vigorously. After 2-3 h, $1.0 \mathrm{~g}$ of $\mathrm{NaNO}_{3}$ and $5.0 \mathrm{~g}$ of $\mathrm{KMnO}_{4}$ were slowly added one by one at low temperatures (0-5 $\left.{ }^{\circ} \mathrm{C}\right)$. The mixture was allowed to warm at room temperature (25 ${ }^{\circ} \mathrm{C}$ ) and stirred for $2 \mathrm{~h}$. Again, the conical flask with the reaction mixture was placed in an ice bath and $110 \mathrm{~mL}$ water was added slowly; the temperature was not raised more than $90{ }^{\circ} \mathrm{C}$ with constant stirring for $30 \mathrm{~min}$. The mixture was poured into $200 \mathrm{~mL}$ of water, after which $7 \mathrm{~mL} 30 \% \mathrm{H}_{2} \mathrm{O}_{2}$ was added through the inner wall of the conical flask. Immediately the color of the solution changed from dark brown to pale yellow. The solution was then filtered using a Millipore filter paper to get a pale yellow residue. The residue was re-dispersed in water, centrifuged at $15000 \mathrm{rpm}$ and washed several times with water until the $\mathrm{pH}$ of the solution became neutral. The resultant solid material was dried in vacuum at room temperature $\left(25^{\circ} \mathrm{C}\right)$ and stored in an ambient environment to get pure GO.

2.3.3 Synthesis of 1,3-bis(2'-benzimidazolyl)-5aminobenzene-grafted reduced GO (GONBZ). First, a GO solution was prepared by ultrasonic exfoliation of graphite oxide (50 $\mathrm{mg})$ into deionized water $(30 \mathrm{~mL})$ for $30 \mathrm{~min}$. Then, 3-bis $\left(2^{\prime}-\right.$ benzimidazolyl)-5-aminobenzene $(50 \mathrm{mg})$ was added into the solution and sonicated for another $15 \mathrm{~min}$. It was heated at $140{ }^{\circ} \mathrm{C}$ for $24 \mathrm{~h}$ in an autoclave, following which the mixture was filtered and washed with methanol ( $25 \mathrm{~mL})$ to remove excess 3bis-(2'-benzimidazolyl)-5-aminobenzene and dried. GONBZ was obtained and then, it was taken in $30 \mathrm{~mL}$ ethanol and hydrazine monohydrate $(3.5 \mathrm{~mL})$ was added dropwise, resulting in a reduced form of GONBZ (RGONBZ), a black residue (35 mg).

2.3.4 In situ synthesis of 1,3-bis(2'-benzimidazolyl)-5aminobenzene-grafted reduced GO-coated polyaniline. The chemically converted graphene oxide (RGONBZ) was dispersed in $1.5(\mathrm{~N}) \mathrm{HCl}$ for $2 \mathrm{~h}$; aniline in $1.5(\mathrm{~N}) \mathrm{HCl}$ solution was added and stirred for $2 \mathrm{~h}$ keeping at room temperature. The ammonium persulfate solution was taken in a water medium and added dropwise to the aniline-containing chemically converted graphene oxide solution; the polymerization started via radical formation. It was stirred overnight and then kept inside a refrigerator for $24 \mathrm{~h}$, resulting in a green residue, which was collected via centrifugation and washed with water and methanol to remove the oligomers. Then, it was dried by keeping inside an oven. Three different concentrations of aniline were taken separately (Table 1 ).

\section{Results and discussion}

\subsection{Spectral study}

First, 1,3-bis(2'-benzimidazolyl)-5-aminobenzene was prepared via a condensation reaction of 5-aminoisophthalic acid and $o$ phenylenediamine. The condensation product was characterized by ${ }^{1} \mathrm{H}$-NMR spectroscopy using DMSO- $\mathrm{d}_{6}$ as the solvent (Fig. S1 $\dagger$ ). In case of 1,3-bis(2'-benzimidazolyl)-5aminobenzene, two types of aromatic proton peaks appeared at $8.13 \mathrm{ppm}(\mathrm{s}, 1 \mathrm{H})$ and $7.48 \mathrm{ppm}(\mathrm{s}, 2 \mathrm{Hs})$, corresponding to the 5 -aminoisophthalic acid moiety. The other two types of aromatic peaks from the benzimidazole moiety appeared at $7.58 \mathrm{ppm}$ (broad spectra, $4 \mathrm{H}$ ) and $7.19 \mathrm{ppm}(\mathrm{dd}, 4 \mathrm{H}, J=2.8 \mathrm{~Hz})$. The FT-IR spectra (Fig. $\mathrm{S} 2 \dagger$ ) revealed that the broad peaks at $3385 \mathrm{~cm}^{-1}$ and $3200 \mathrm{~cm}^{-1}$ corresponded to free $\mathrm{N}-\mathrm{H}$ and hydrogen-bonded $\mathrm{N}-\mathrm{H}$; also, a new band at $1627 \mathrm{~cm}^{-1}(\mathrm{C}=\mathrm{N})$ and the absence of carbonyl stretching frequency $\left(1700 \mathrm{~cm}^{-1}\right)$ implied the proper formation of 1,3-bis(2'-benzimidazolyl)-5aminobenzene. Subsequently, RGONBZ and RGONBZ_PANI were prepared using the synthesized GO.

The FT-IR spectra of GO and RGONBZ were recorded and the most characteristic absorption band of GO appeared at $1724 \mathrm{~cm}^{-1}$, corresponding to the $\mathrm{C}=\mathrm{O}$ stretching mode of carboxyl groups on the GO sheets; other relevant peaks appeared at $1622 \mathrm{~cm}^{-1}$ for epoxide groups and at $1380 \mathrm{~cm}^{-1}$ for skeletal ring vibrations (Fig. 1a). The broad bands at 3450$3600 \mathrm{~cm}^{-1}$ implied the presence of tertiary $\mathrm{C}-\mathrm{OH}$ groups at the
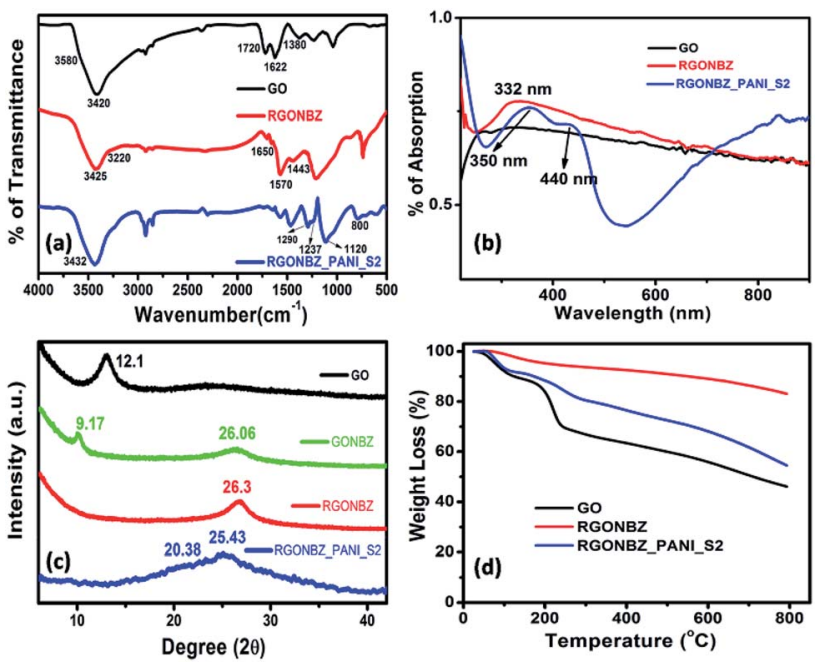

Fig. 1 (a) FT-IR spectra, (b) UV-vis spectra, (c) XRD patterns and (d) TGA curves of GO, RGONBZ and RGONBZ_PANI_S2.

Table 1 Amounts of RGONBZ, aniline and APS in three different sets of binary composites

\begin{tabular}{lllll}
\hline & $\begin{array}{l}\text { RGONBZ in } 20 \mathrm{~mL} \\
\text { Materials }\end{array}$ & $\begin{array}{l}\text { Aniline in } 20 \mathrm{~mL} \\
1.5(\mathrm{~N}) \mathrm{HCl}\end{array}$ & $\begin{array}{l}\text { APS in } 10 \mathrm{~mL} \\
\text { water }\end{array}$ & $\begin{array}{l}\text { RGONBZ to aniline } \\
\text { ratio }\end{array}$ \\
\hline RGONBZ_PANI_S1 & $10 \mathrm{mg}$ & $102 \mathrm{mg}$ & $20 \mathrm{mg}$ & $1: 10.2$ \\
RGONBZ_PANI_S2 & $10 \mathrm{mg}$ & $204 \mathrm{mg}$ & $40 \mathrm{mg}$ & $1: 20.4$ \\
RGONBZ_PANI_S3 & $10 \mathrm{mg}$ & $408 \mathrm{mg}$ & $80 \mathrm{mg}$ & $1: 40.8$
\end{tabular}


edge of the GO sheets. However, after functionalization and reduction, the peak at $1724 \mathrm{~cm}^{-1}$ disappeared due to the absence of the carboxylic group. In the spectrum of RGONBZ, new peaks at 1570 and $1443 \mathrm{~cm}^{-1}$ corresponded to the benzene stretching frequencies; besides, small peaks at $1650 \mathrm{~cm}^{-1}$ were attributed to the $\mathrm{C}=\mathrm{N}$ bond stretching vibration of the imidazole part. The most significant benzimidazole functional group-containing free and hydrogen-bonded $\mathrm{N}-\mathrm{Hs}$ exhibited peaks at $3425 \mathrm{~cm}^{-1}$ and $3220 \mathrm{~cm}^{-1}$, respectively. For the RGONBZ_PANI composites, a sharp band at $1570 \mathrm{~cm}^{-1}$ corresponded to the $\mathrm{C}=\mathrm{N}$ stretching displacement of the quinoid structure of polyaniline. The peaks at $1290 \mathrm{~cm}^{-1}$ and $1237 \mathrm{~cm}^{-1}$ were attributed to the $\mathrm{C}-\mathrm{N}$ stretching vibration of the secondary aromatic amine group and $\mathrm{C}-\mathrm{N}^{*+}$ in the acid-doped PANI, respectively. The $\mathrm{C}=\mathrm{N}$ stretching vibration resulted in a peak at $1123 \mathrm{~cm}^{-1}$. The remaining peak at $800 \mathrm{~cm}^{-1}$ was attributed to the aromatic ring out-of-plane bending vibrations of the $\mathrm{C}-\mathrm{H}$ bond.

\subsection{Absorption study}

The UV-vis absorption spectra of the ethanol-dispersed GO, RGONBZ and RGONBZ_PANI_S2 composites are shown in Fig. 1b. GO showed two absorption peaks: the lower one was at the 230-240 $\mathrm{nm}$ region for the $\pi-\pi^{*}$ transition of the aromatic $\mathrm{C}-\mathrm{C}$ bond; comparatively, the higher one contained a broad shoulder at $310 \mathrm{~nm}$ assigned to the $\mathrm{n}-\pi^{*}$ transition of $\mathrm{C}=\mathrm{O}$ bonds. The absorption maxima at $332 \mathrm{~nm}$ indicated the presence of a benzimidazole moiety in RGONBZ, which appeared in the $350 \mathrm{~nm}$ region for the RGONBZ_PANI_S2 composite as the benzimidazole peak merged with the $\pi-\pi *$ electron transition of the benzenoid rings of the polyaniline nanostructure. Two other absorption peaks at $440 \mathrm{~nm}$ and $850 \mathrm{~nm}$ appeared in the usual position for the polaron $-\pi^{*}$ transition and $\pi$-polaron transition, respectively.

\subsection{XRPD and TGA study}

Fig. 1c shows the XRPD patterns of GO, RGONBZ and binary composite RGONBZ_PANI_S2. In the synthesized GO, a strong signal appeared at $2 \theta=12.1^{\circ}$, corresponding to an interlayer distance of $7.73 \AA$ of the GO sheet due to the intercalation of the nitrogen-containing functional groups on the graphite sheets; a signal was observed at $2 \theta=23^{\circ}$ for multilayer graphite..$^{30,31}$ After functionalization, GONBZ (before reduction by treating with hydrazine monohydrate) exhibited two main peaks. One was observed at a lower $2 \theta$ region $\left(2 \theta=9.17^{\circ}\right)$, demonstrating the presence of a benzimidazole moiety on the graphite sheets, resulting in an increased interlayer distance between the sheets. The other was observed at $2 \theta=26.06^{\circ}$, which represented chemically converted graphene. The diffraction of all the three binary composites containing polyaniline coated with chemically modified reduced graphene oxide provided information about the interaction of RGONBZ with polyaniline besides its slightly crystalline nature in the composites. ${ }^{32}$ The peaks at $2 \theta=$ $20.38^{\circ}(100)$ and $25.43^{\circ}$ (110) appeared due to the repeating nature of parallel and perpendicular polyaniline chains, respectively (Fig. S3†). Both the synthesized chemically modified reduced graphene oxide (RGONBZ) and its polyaniline-coated binary composite (RGONBZ_PANI_S2) were checked for thermal stability (Fig. 1d). We found that the benzimidazole-grafted material RGONBZ has better stability than the binary composite material. Up to $500{ }^{\circ} \mathrm{C}$, RGONBZ decomposed by only $10 \%$, whereas the binary material decomposed by $28 \%$.

\subsection{XPS study}

The chemical composition and the bonding nature of the atoms in the chemically converted reduced graphene oxide as well as its binary composite containing polyaniline were further characterized by XPS analysis. The set of peaks at 285, 400 and $532.5 \mathrm{eV}$ for both RGONBZ and the composite material (RGONBZ_PANI_S2) in the wide-scan XPS spectra confirmed the presence of $\mathrm{C}, \mathrm{N}$ and $\mathrm{O}$, respectively (Fig. 2a and 3a). Also, the presence of $\mathrm{N}$ indicated the proper transformation from $\mathrm{GO}$ to $\mathrm{N}$-doped chemically modified reduced GO. For comparison, XPS studies of pure GO were performed and it was observed that the signal for $\mathrm{N}$ was absent. The XPS spectra of GO as well as the deconvoluted $\mathrm{C}$ 1s spectra and $\mathrm{O}$ 1s spectra of GO have been presented in Fig. S4. $\dagger$

Furthermore, the deconvoluted spectra (Fig. 2b-d) of the 1s orbitals of $\mathrm{C}, \mathrm{N}$ and $\mathrm{O}$ provide detailed information about the bonding nature of these atoms. The enlarged scan of $\mathrm{N} 1 \mathrm{~s}$ of binary composites (Fig. 3c) of polyaniline-containing chemically modified reduced GO (RGONBZ_PANI_2) could be deconvoluted into three sub-peaks at $399,399.7$ and $401.4 \mathrm{eV}$, corresponding to imine $(-\mathrm{N}=)$, amine $(-\mathrm{NH}-)$ and protonated amine $\left(-\mathrm{N}^{{ }^{+}-}\right) .{ }^{33,34}$ The high-resolution $\mathrm{C}$ 1s spectra (Fig. 3b) could be deconvoluted into three sub-peaks at 284.15, 285.3 and $288.4 \mathrm{eV}$ for $\mathrm{C}=\mathrm{C} / \mathrm{C}-\mathrm{C}, \mathrm{C}-\mathrm{N} / \mathrm{C}-\mathrm{O}$ and $\mathrm{C}=\mathrm{O}$, respectively, concomitant with the formation of PANI. ${ }^{31}$ The deconvoluted spectra of $\mathrm{O} 1 \mathrm{~s}$ (Fig. 3d) of RGONBZ_PANI_S2 exhibited proper correlation with
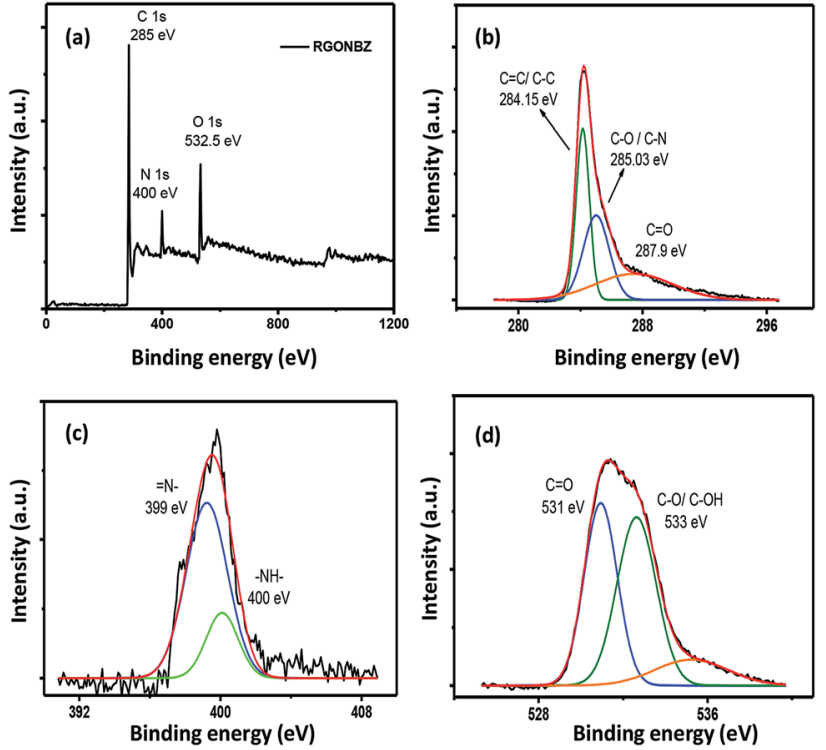

Fig. 2 XPS study of RGONBZ: (a) whole scan; deconvoluted spectra of (b) $\mathrm{C} 1 \mathrm{~s}$, (c) $\mathrm{N} 1 \mathrm{~s}$ and (d) $\mathrm{O} 1 \mathrm{~s}$. 

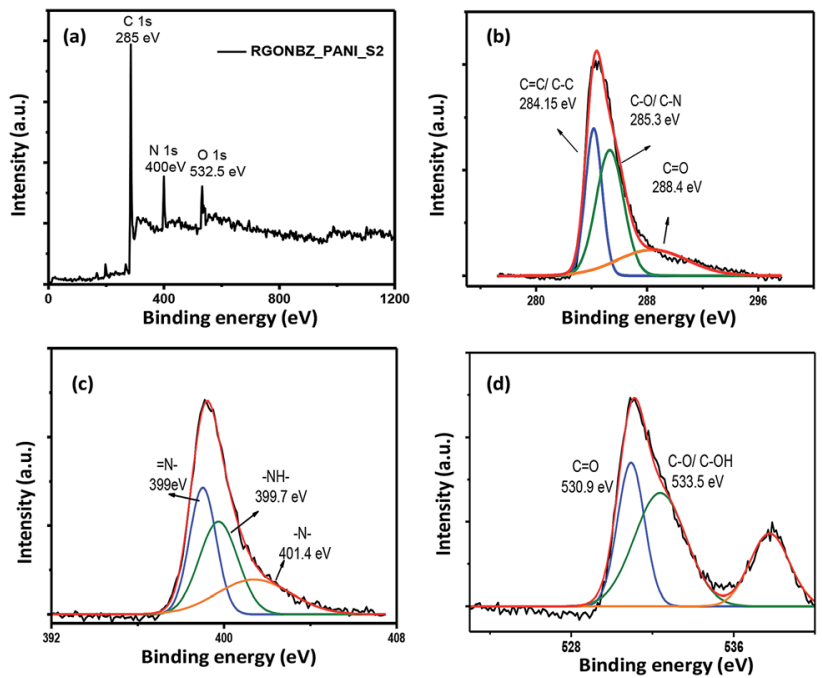

Fig. 3 XPS study of RGONBZ_PANI_S2: (a) whole scan; deconvoluted spectra of (b) C 1s, (c) N 1s and (d) $O 1 \mathrm{~s}$.

its oxygen-containing functional groups. The appearance of a peak at $536 \mathrm{eV}$ (Fig. 2d) or at $538 \mathrm{eV}$ (Fig. 3d) was possibly owing to the sodium Auger peak (Na KLL).

\subsection{Morphological study}

FESEM and TEM are two effective tools for the morphological interpretation of electrode materials. The morphologies are shown in Fig. 4. The synthesized GO showed wrinkled morphology, i.e., a layer-like structure with a lateral dimension up to several micrometers (Fig. S5†). Fig. 4a and c show the FESEM and TEM images of RGONBZ, respectively, revealing that the layered structure can be maintained in the chemically functionalized reduced graphene oxide with less aggregation, which implied the effectiveness of functionalization. In case of binary composites, randomly placed polyaniline nanorods
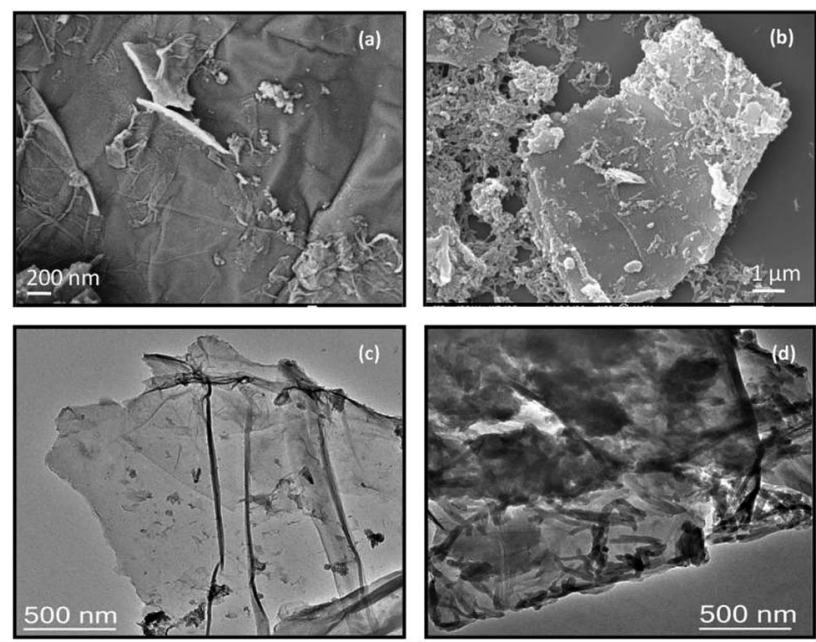

Fig. 4 FESEM images of (a) RGONBZ and (b) RGONBZ_PANI_S2. TEM images of (c) RGONBZ and (d) RGONBZ_PANI_S2. along with some stacked nanorod clusters were observed on the RGONBZ sheets (Fig. 4b), which might be explained by the nucleation mechanism occurring during the polymerization process. The complete coverage was further confirmed by the TEM image (Fig. 4d). The dimensions of the nanorods were $300-400 \mathrm{~nm}$ length and $25-30 \mathrm{~nm}$ diameter. The presence of a basic benzimidazole group on the surface of graphene sheets determined the polymerization nature of anilinium ions (protonated aniline monomers). The electrostatic attraction between these two inverse variants makes the polymerization process random and heterogeneous.

\subsection{Electrochemical study}

The electrochemical energy storage properties of RGONBZ and the three sets of RGONBZ_PANI binary composites were analyzed by cyclic voltammetry (CV) and the galvanostatic charge-discharge (GCD) method using three-electrode cell systems in a standard aqueous $1 \mathrm{M} \mathrm{H}_{2} \mathrm{SO}_{4}$ solution as the electrolyte. The cyclic voltammograms of chemically modified reduced graphene oxide, i.e., RGONBZ in the potential window from $-0.4 \mathrm{~V}$ to $0.6 \mathrm{~V}(v s$. $\mathrm{AgCl} / \mathrm{Ag})$ are shown in Fig. $5 \mathrm{a}$, and the representative charge-discharge curves at different current densities are shown in Fig. 5b. For RGONBZ, two oxidation peaks were observed at $0.248 \mathrm{~V}$ and $0.394 \mathrm{~V}$ and the corresponding reduction peaks were observed at $0.113 \mathrm{~V}$ and $0.312 \mathrm{~V}$ in $1 \mathrm{M} \mathrm{H}_{2} \mathrm{SO}_{4}$ solution during the forward (anodic) and backward (cathodic) sweeps at a scan rate of $30 \mathrm{mV} \mathrm{s}^{-1}$ (Fig. S6a†), suggesting the presence of pseudocapacitive features. The binary material RGONBZ_PANI comprising polyaniline and the chemically modified reduced graphene oxide (RGONBZ) exhibited the same pair of oxidation and reduction peaks but at different locations. Typically, the oxidation peaks were located at $0.07 \mathrm{~V}, 0.42 \mathrm{~V}$ and $0.64 \mathrm{~V}$ and their corresponding reduction peaks were observed at $0.18 \mathrm{~V}, 0.44 \mathrm{~V}$ and $0.66 \mathrm{~V}$ (Fig. S6b $\dagger$ ) in $1 \mathrm{M} \mathrm{H}_{2} \mathrm{SO}_{4}$ solution.
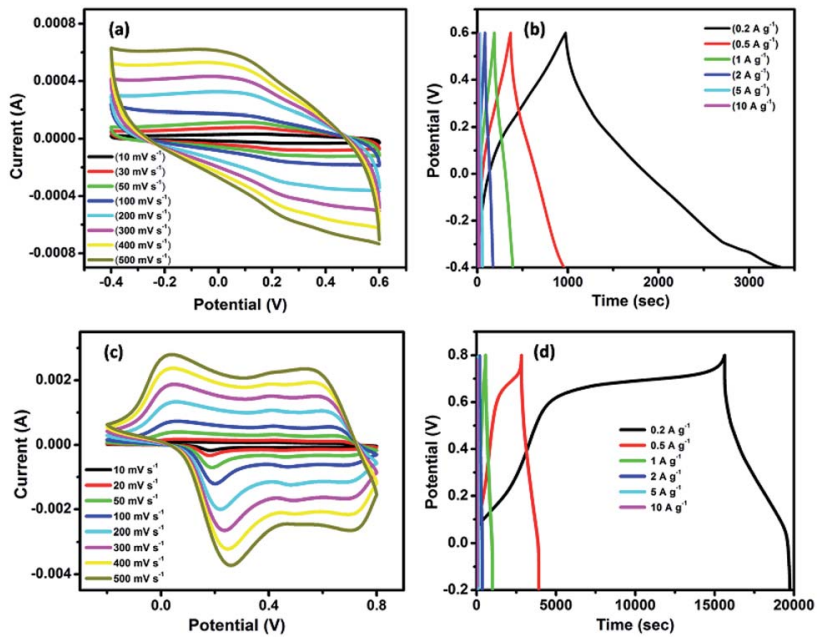

Fig. 5 (a) CV study; (b) GCD plot for RGONBZ in $1 \mathrm{M} \mathrm{H}_{2} \mathrm{SO}_{4}$; (c) $\mathrm{CV}$ study for RGONBZ_PANI_S2 in $1 \mathrm{M} \mathrm{H}_{2} \mathrm{SO}_{4}$ solution at various scan rates from $10 \mathrm{mV} \mathrm{s}^{-1}$ to $500 \mathrm{mV} \mathrm{s}^{-1}$; (d) GCD plots obtained at different current densities (0.2-10) $\mathrm{A} \mathrm{g}^{-1}$ for RGONBZ_PANI_S2. 
At a high scan rate, the CV curve was nearly rectangular in shape due to the charge propagation within the electrode. The CV curve (Fig. 5c) at a higher scan rate manifested the capacitive response of RGONBZ_PANI from the combination of EDLC and pseudo-capacitance. ${ }^{35}$ The presence of a heteroatom (nitrogen) in RGONBZ and polyaniline on the surface of the binary composite RGONBZ_PANI promoted the redox reactions, indicating the pseudo-capacitance nature of our electrode materials. The charge-discharge property was perceived in the same solution and the longest discharging duration was acquired than that of RGONBZ. The high-performance energy storage material stipulated not only low internal resistivity but also less energy waste during the charge-discharge process. The longest rate capabilities obtained for RGONBZ_PANI_S2 were evaluated by discharging processes at different current densities starting from $0.2 \mathrm{~A} \mathrm{~g}^{-1}$ to $10 \mathrm{~A} \mathrm{~g}^{-1}$ and the maximum specific capacitance achieved was $823 \mathrm{~F} \mathrm{~g}^{-1}$ at $0.2 \mathrm{~A} \mathrm{~g}^{-1}$ current density (Fig. 5d); for RGONBZ, it was $477 \mathrm{~F} \mathrm{~g}^{-1}$ (Fig. 5b).

The high electrical conductivity of polyaniline facilitated the charge diffusion and transfer during the charge-discharge process, which displayed much higher specific capacitance in the binary composite (RGONBZ_PANI_S2) than that in RGONBZ alone. ${ }^{31}$ Other sets of binary composites, i.e., RGONBZ_PANI_S1 and RGONBZ_PANI_S3 exhibited specific capacitances of 356 F $\mathrm{g}^{-1}$ and $484.4 \mathrm{~F} \mathrm{~g}^{-1}$, respectively, at $0.2 \mathrm{~A} \mathrm{~g}^{-1}$ current density. The charge-discharge cycles of the binary mixtures RGONBZ_PANI_S1 and RGONBZ_PANI_S3 at different current densities are depicted in Fig. S8a and b. $\dagger$ In Fig. S8c, $\uparrow$ variations in the specific capacitance with current densities of all three binary composites are shown. The plot of peak current $v s$. scan rate for RGONBZ and RGONBZ_PANI_S2 was obtained from the CV curve and is depicted in Fig. S9. $\dagger$ The slope of the line was also calculated from the plot.

The maximum energy density obtained for RGONBZ_PANI_S2 was $114.4 \mathrm{~W} \mathrm{~h} \mathrm{~kg}^{-1}$ at $0.2 \mathrm{~A} \mathrm{~g}^{-1}$ current density, and the value for RGONBZ was $66.25 \mathrm{~W} \mathrm{~h} \mathrm{~kg}{ }^{-1}$; the corresponding power density values were same for both electrode materials $\left(100.0 \mathrm{~W} \mathrm{~kg}^{-1}\right)$. Fig. $\mathrm{S} 7 \dagger$ exhibits the variation in the specific capacitance of RGONBZ and the most effective binary mixture (RGONBZ_PANI_S2) with current density. Additionally, as electrodes with great expediency, the cyclic stabilities of both materials were tested up to 5000 cycles, and the capacitance retention value for the reduced material (RGONBZ) was $87.9 \%$ at $10 \mathrm{~A} \mathrm{~g}^{-1}$. The binary composite material RGONBZ_PANI_S2 maintained specific capacitance of $77.5 \%$ of its initial value at $20 \mathrm{~A} \mathrm{~g}^{-1}$ current density, indicating a pronounced pseudocapacitive response (Fig. 6). The calculated value of specific capacitance and the retention value of binary composites were compared with those of different functional rGO materials and PANI composites (Table S1†). The electrochemical impedance spectrum was well defined using the Nyquist plot, i.e., the plot of the imaginary part $\left(Z^{\prime \prime}\right)$ of the impedance against the real part $\left(Z^{\prime}\right)$ could be characterized by two different geometries: a semicircle at high frequency and a straight line at low frequency (Fig. S10 $\dagger$ ). The equivalent circuit diagram was also constructed to fit the Nyquist plots (Fig. S11 $\dagger$ ). This supported the relatively

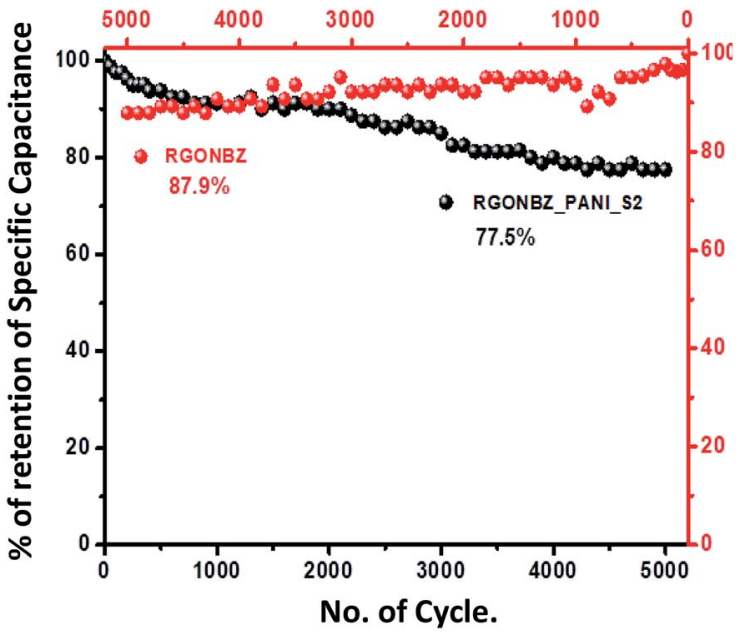

Fig. 6 Charge-discharge cycle stability plots of RGONBZ (red) and RGONBZ_PANI_S2 (black).

higher storage capacity of RGONBZ_PANI_S2 than that of RGONBZ.

\section{Conclusions}

Chemically converted reduced graphene oxide (RGONBZ) and three sets of binary composites containing conductive polymer polyaniline and the reduced chemically converted graphene oxide (RGONBZ_PANI_S1, S2 and S3) were successfully synthesized and characterized. All materials were characterized by FTIR spectroscopy and XPS. Their structures were studied by various techniques such as XRD and TGA and by various morphological analyses such as FESEM and TEM. Electrochemical measurements were recorded for all the materials. Cyclic voltammetry of 1,3-bis(2'-benzimidazolyl)-5aminobenzene-grafted reduced graphene oxide (RGONBZ) indicated the pseudo-capacitive nature; the galvanostatic charge-discharge experiment provided the specific capacitance value of $477 \mathrm{~F} \mathrm{~g}^{-1}$ at $0.2 \mathrm{~A} \mathrm{~g}^{-1}$ current density. Similar studies were performed for polyaniline-coated RGONBZ materials (RGONBZ_PANI_S1, S2 and S3), which also indicated the pseudo-capacitance nature; the best specific capacitance value of $823 \mathrm{~F} \mathrm{~g}^{-1}$ was obtained at $0.2 \mathrm{~A} \mathrm{~g}^{-1}$ current density for RGONBZ_PANI_S2. The cyclic stability retention for RGONBZ was $87.9 \%$ at $10 \mathrm{~A} \mathrm{~g}^{-1}$ current density and the value for the polyaniline-coated material RGONBZ_PANI_S2 was $77.5 \%$ at $20 \mathrm{~A} \mathrm{~g}^{-1}$ current density, which indicated the pseudocapacitance nature.

\section{Conflicts of interest}

There are no conflicts to declare.

\section{Acknowledgements}

A. Roy thanks to CSIR, New Delhi, India for the fellowship. Dr Dhibar is thankful to SERB-DST, Government of India, for the 
National Post-Doctoral Fellowship (N-PDF) [File No. PDF/2016/ 002589]. Dr S. Malik acknowledges CSIR, India (project no. 01(2875)/17/EMR-II) for financial support.

\section{References}

1 L. L. Zhang and X. S. Zhao, Chem. Soc. Rev., 2009, 38, 25202531.

2 X.-L. Wu, T. Wen, H.-L. Guo, S. Yang, X. Wang and A.-W. Xu, ACS Nano, 2013, 7, 3589-3597.

3 K. T. Lee, J. C. Lytle, N. S. Ergang, S. M. Oh and A. Stein, Adv. Funct. Mater., 2005, 15, 547-556.

4 H. Jiang, P. S. Lee and C. Li, Energy Environ. Sci., 2013, 6, 4153.

5 Y. Wang, Z. Shi, Y. Huang, Y. Ma, C. Wang, M. Chen and Y. Chen, J. Phys. Chem. C, 2009, 113, 13103-13107.

6 M. D. Stoller, S. Park, Y. Zhu, J. An and R. S. Ruoff, Nano Lett., 2008, 8, 3498-3502.

7 Z. Sun, D. K. James and J. M. Tour, J. Phys. Chem. Lett., 2011, 2, 2425-2432.

8 G. Wang, J. Yang, J. Park, X. Gou, B. Wang, H. Liu and J. Yao, J. Phys. Chem. C, 2008, 112, 8192-8195.

9 P. Simon and Y. Gogotsi, Nat. Mater., 2008, 7, 845-854.

10 D. Wang, D. Choi, J. Li, Z. Yang, Z. Nie, R. Kou, D. Hu, C. Wang, L. V. Saraf and J. Zhang, ACS Nano, 2009, 3, 907914.

11 Y. Huang, J. Liang and Y. Chen, Small, 2012, 8, 1805-1834.

12 J. Shen, Y. Hu, C. Li, C. Qin, M. Shi and M. Ye, Langmuir, 2009, 25, 6122-6128.

13 S. Park and R. S. Ruoff, Nat. Nanotechnol., 2009, 4, 217-224.

14 D. Yu and L. Dai, J. Phys. Chem. Lett., 2010, 1, 467-470.

15 S. Bai and X. Shen, RSC Adv., 2012, 2, 64-98.

16 W. Chen, S. Li, C. Chen and L. Yan, Adv. Mater., 2011, 23, 5679-5683.

17 B. Xu, S. Yue, Z. Sui, X. Zhang, S. Hou, G. Cao and Y. Yang, Energy Environ. Sci., 2011, 4, 2826-2830.

18 Q. Wu, Y. Sun, H. Bai and G. Shi, Phys. Chem. Chem. Phys., 2011, 13, 11193-11198.
19 Y. Lu, F. Zhang, T. Zhang, K. Leng, L. Zhang, X. Yang, Y. Ma, Y. Huang, M. Zhang and Y. Chen, Carbon, 2013, 63, 508-516. 20 W. Ai, W. Zhou, Z. Du, Y. Du, H. Zhang, X. Jia, L. Xie, M. Yi, T. Yu and W. Huang, J. Mater. Chem., 2012, 22, 23439-23446.

21 X.-M. Feng, R.-M. Li, Y.-W. Ma, R.-F. Chen, N.-E. Shi, Q.-L. Fan and W. Huang, Adv. Funct. Mater., 2011, 21, 2989-2996.

22 S. Liu, X. Liu, Z. Li, S. Yang and J. Wang, New J. Chem., 2011, 35, 369-374.

23 C. Basavaraja, W. J. Kim, Y. Do Kim and S. H. Do, Mater. Lett., 2011, 65, 3120-3123.

24 L. Mao, K. Zhang, H. S. O. Chan and J. Wu, J. Mater. Chem., 2012, 22, 80-85.

25 R. Wang, M. Han, Q. Zhao, Z. Ren, X. Guo, C. Xu, N. Hu and L. Lu, Sci. Rep., 2017, 7, 44562.

26 G. Xu, N. Wang, J. Wei, L. Lv, J. Zhang, Z. Chen and Q. Xu, Ind. Eng. Chem. Res., 2012, 51, 14390-14398.

27 Y. Liu, R. Deng, Z. Wang and H. Liu, J. Mater. Chem., 2012, 22, 13619-13624.

28 L. Fu, M. Pan, Y.-H. Li, H.-B. Wu, H.-P. Wang, C. Yan, K. Li, S.-C. Wei, Z. Wang and C.-Y. Su, J. Mater. Chem., 2012, 22, 22496-22500.

29 W. S. Hummers Jr and R. E. Offeman, J. Am. Chem. Soc., 1958, 80, 1339.

30 Y. Li, X. Zhao, P. Yu and Q. Zhang, Langmuir, 2013, 29, 493500.

31 S. Mondal, U. Rana and S. Malik, Chem. Commun., 2015, 51, 12365-12368.

32 G. Wang, X. Shen, B. Wang, J. Yao and J. Park, Carbon, 2009, 47, 1359-1364.

33 D. C. Marcano, D. V. Kosynkin, J. M. Berlin, A. Sinitskii, Z. Sun, A. Slesarev, L. B. Alemany, W. Lu and J. M. Tour, ACS Nano, 2010, 4, 4806-4814.

34 N. Wu, X. She, D. Yang, X. Wu, F. Su and Y. Chen, J. Mater. Chem., 2012, 22, 17254-17261.

35 L. Sun, L. Wang, C. Tian, T. Tan, Y. Xie, K. Shi, M. Li and H. Fu, RSC Adv., 2012, 2, 4498-4506. 